\section{Las prácticas sociocomunitarias en la Universidad Nacional de Río Cuarto. Un estudio evaluativo}

\author{
Viviana Macchiarola \\ macchiarolav@gmail.com \\ (iD) orcid.org/0000- 0001- 6951- 4892 \\ Ana Lucía Pizzolitto \\ alpizzolitto19@yahoo.com.ar \\ (iD) orcid.org/0000-0002-4935- 5019
}

\author{
Verónica Pugliese Solivellas \\ veronicaluciapugliese@gmail.com \\ (iD) orcid.org/0000- 0002- 9959- 0404 \\ Universidad Nacional de Río Cuarto, \\ Argentina
}

Sujetos y relaciones en extensión universitaria / Desafíos de gestión

다(1)(2)(2)
RECEPCIÓN: 06/04/20

ACEPTACIÓN FINAL: 16/05/20

\section{Resumen}

En este este artículo se presentan resultados parciales obtenidos en una investigación evaluativa e interpretativa desarrollada en la Universidad Nacional de Río Cuarto. El objeto del análisis es un programa institucional que propone la incorporación de las prácticas sociocomunitarias a los planes de estudio. Las mismas suponen una ruptura epistemológica en tanto constituyen una nueva forma de conocer, alternativa a los regímenes tradicionales de verdad, ciencia y universidad y el compromiso social de la universidad. El objetivo del estudio es valorar los procesos y resultados emergentes en estas prácticas desde las perspectivas de todos quienes se involucran en ellas: docentes, estudiantes y actores de la comunidad. El propósito es brindar conocimiento que oriente las decisiones políticas y las acciones de gestión universitaria. Puntualizamos en este artículo las concepciones acerca de las prácticas sociocomunitarias y los aportes que estas hacen a la comunidad desde la perspectiva de los propios actores.

Palabras clave: extensión universitaria prácticas sociocomunitarias; investigación; evaluación; aprendizaje
Socio-community practices at the National University of Río Cuarto. An evaluative study

\section{Abstract}

The partial results obtained in an evaluative and interpretive research conducted at the National University of Río Cuarto are displayed in this paper. The object of the study is an institutional program that proposes the incorporation of socio- community practices into the curriculum. They represent an epistemological rupture as they constitute a new way of knowing, which is alternative to the traditional regimes of truth, science and university and the social commitment of the university. The objective of the study is to assess the processes and emerging outcomes in these practices, from the perspectives of all who are involved in them: teachers, students and community actors. The purpose is to provide knowledge that guides political decisions and university management actions. We point out in this article, the conceptions about the practical and the contributions that they make to the community, from the perspective of the actors themselves.

Keywords: university extension; sociocommunity practices; investigation; evaluation; learning

\author{
Práticas sócio-comunitárias na \\ Universidade Nacional de Río Cuarto. \\ Um estudo avaliativo
}

\section{Resumo}

No presente artigo se apresentam resultados parciais obtidos em uma pesquisa avaliativa e interpretativa realizada na Universidade Nacional de Río Cuarto. O objeto do estudo é um programa institucional que propõe a incorporação de práticas sociocomunitárias aos planos curriculares. Elas representam uma ruptura epistemológica, pois constituem uma nova maneira de conhecer, alternativa aos regimes tradicionais da verdade, ciência e universidade e do compromisso social da universidade. O objetivo do estudo é avaliar os processos e resultados emergentes dessas práticas, a partir das perspectivas de todos os envolvidos nelas: professores, alunos e atores da comunidade. O objetivo é oferecer conhecimento para orientar decisões políticas e ações de gestão universitária. Neste artigo, destacamos as concepções acerca das práticas e das contribuições que eles fazem para a comunidade, desde a perspectiva dos próprios atores.

Palavras-chave: extensão universitária; práticas sociocomunitárias; pesquisa; avaliação; aprendizagem

Para citación de este artículo: Macchiarola, V.; Pizzolitto, A. L. y Pugliese Solivellas, V. (2020). Las prácticas sociocomunitarias en la Universidad Nacional de Río Cuarto. Un estudio evaluativo. +E: Revista de Extensión Universitaria, 10(12.Ene-Jun), 1-19. doi: 10.14409/extension.v10i12.EneJun.8967. 


\section{Introducción}

Este artículo presenta una investigación evaluativa del proyecto de incorporación de prácticas sociocomunitarias al currículo que se desarrolla en la Universidad Nacional de Río Cuarto (UNRC). Esta institución incorpora, desde el año 2009, prácticas sociocomunitarias (PSC) a los planes de estudio en el marco del desarrollo de su Plan Estratégico Institucional y de uno de sus objetivos estratégicos: "Compromiso social mediante la construcción de redes territoriales".

El objetivo general de esta iniciativa es "construir y afianzar un currículo que coadyuve a la creación de conciencia social y ciudadana, en el marco de una función crítica de la Universidad" (Resolución CS №322/09). En estas prácticas, los estudiantes aprenden los contenidos de las asignaturas correspondientes a la vez que realizan junto a la comunidad proyectos que contribuyan a la comprensión y resolución de problemas del territorio.

Para concretar esta decisión política, desde la Secretaría de Planeamiento y Relaciones Institucionales de Rectorado se realizaron dos convocatorias para la presentación, evaluación y desarrollo de PSC. La primera se efectuó en septiembre de 2015 y permitió implemen$\operatorname{tar} 48$ proyectos en las cinco facultades que involucraron a 29 carreras ( $63 \%$ de las carreras de la UNRC), 122 espacios curriculares y 231 profesores (12\% del claustro docente).

En el marco de la Convocatoria "Sinergias", llevada a cabo por la SPU (Secretaría de Políticas Universitarias) del Ministerio de Educación y Deportes de la Nación, se hizo una segunda convocatoria (año 2017) que posibilitó fortalecer el desarrollo de las PSC, extender su incorporación a nuevas carreras de la UNRC, y promover así un mayor ajuste entre los problemas vividos y sentidos por la comunidad y las prácticas de aprendizaje y extensión que realiza la universidad, en tanto que ayudó a la difusión y visibilización del proyecto. Como resultado de esa Convocatoria se desarrollan 40 nuevas PSC en las cinco facultades, que involucraron a 52 carreras de las 56 que ofrece la UNRC, 126 asignaturas y 83 instituciones y organizaciones sociales.

Actualmente, se encuentra en marcha una tercera convocatoria que intenta fortalecer la conformación de redes territoriales y curriculares. Estas redes buscan articular PSC en un mismo territorio y/o en el currículo de una misma carrera y, a la vez, pretenden integrar las PSC con otros proyectos como, por ejemplo, los de investigación, extensión, y talleres del Programa Universidad Barrial.

El avance en el desarrollo de este proyecto interpeló a la UNRC a efectuar una investigación evaluativa del mismo con el objetivo general de valorar sus procesos y resultados desde las perspectivas de todos sus implicados. La responsabilidad educativa y social que supone esta iniciativa requiere estar atentos a sus fortalezas y debilidades para profundizar las primeras y superar las segundas. El propósito de la investigación fue, entonces, brindar conocimiento que informara a las decisiones de política institucional tendientes a la mejora, profundización y extensión del proyecto de incorporación curricular de las PSC.

En este artículo circunscribimos la presentación de los resultados referidos a solo dos objetivos específicos del proyecto de investigación evaluativa: a) comprender las concepciones de los actores participantes sobre las PSC; b) analizar los aportes que las PSC hacen a la comunidad desde la perspectiva de sus participantes. 


\section{Contexto conceptual}

La investigación evaluativa requiere de marcos teóricos que actúen a manera de referentes o criterios para emitir juicios de valor sobre el objeto evaluado. Ellos explicitan las posturas del investigador desde las cuales construye y valora su objeto de estudio. Exponemos a continuación los principales fundamentos conceptuales que sustentan el proyecto de PSC en la UNRC y que, por lo tanto, orientan el análisis, interpretación y valoración de los datos empíricos.

\section{Fundamentos epistemológicos}

Las PSC suponen una ruptura epistemológica en tanto constituyen una nueva forma de conocer, alternativa a los regímenes tradicionales de verdad, ciencia y universidad. En este sentido, situaríamos a las PSC en el marco de una epistemología del sujeto conocido, en términos de Vasilachis (2007a, 2007b).

La epistemología del sujeto cognoscente está centrada en el actor que conoce, en su mirada teórica y en su instrumental metodológico, y aborda a su objeto en una relación unidireccional. Si bien en el paradigma interpretativo la distancia entre sujeto y objeto disminuye, el conocimiento sigue centralizado en el sujeto cognoscente y el conocimiento producido y no en la génesis o fuente de sus datos. La epistemología del sujeto conocido, en cambio, se resiste a considerar como objeto al sujeto que forma parte del proceso de conocimiento y lo concibe como sujeto esencialmente igual al sujeto cognoscente. Desde esta perspectiva, el conocimiento requiere de una interacción comunicativa entre el que conoce y el que es conocido, donde ambos sujetos se transforman. Los dos, con igual capacidad para conocer, interactúan y así amplían sus conocimientos acerca del otro, de sí mismo y de la relación cognitiva. Esto implica una construcción cooperativa del conocimiento donde la voz del sujeto conocido no se tergiversa o desaparece bajo los códigos y reglas del discurso científico legitimado. Dice Vasilachis:

"Este sujeto conocido activo y no pasivo, como siendo y haciendo, no como estando y aceptando, como produciendo conocimiento, no como proveyendo de datos útiles para que otros conozcan, ni considerado solo como un depósito de esos datos es el que marca la diferencia entre una epistemología centrada en el sujeto cognoscente y otra, la que propongo, centrada en el sujeto conocido". (2017a, p. 9)

En un sentido similar, y desde la perspectiva de la Investigación-Acción Participativa, Heron y Reason (1997) refieren a una epistemología extendida en la cual quien conoce participa de lo conocido articulando el mundo mediante cuatro tipo de conocimientos que consideramos también se integran en la PSC: 1) un conocimiento experiencial que supone una relación directa, cara a cara con el otro, conociendo mediante la participación empática en las situaciones de vida; 2) un conocimiento representacional, que se basa en el experiencial y lo representa simbólicamente en forma de artes gráficas, plásticas, musicales, vocales y verbales; 3) un conocimiento proposicional o declarativo que se expresa en conceptos, declaraciones y teorías; 4) un conocimiento práctico o saber hacer y saber cómo, que requiere comprensión conceptual de los procedimientos prácticos, representación de los mismos y base experiencial en la situación en la que ocurre la acción. Es decir que articula los tres tipos de conocimientos anteriores en la acción intencional. Las PSC implican un conocimiento práctico, ya que estudiantes, docentes y actores de la comunidad realizan cooperativamente 
acciones intencionales para abordar problemas prácticos, lo que supone un conocer mediante una experiencia directa que suele ser representada de diversas formas no convencionales y que requiere, y a su vez produce, conocimiento proposicional.

\section{Fundamentos pedagógicos}

Otro concepto clave para interpretar las PSC es el de aprendizaje experiencial. Se trata de un aprendizaje situado, en contexto, producto de la acción sobre él. El estudiante aprende participando en prácticas con la comunidad, haciendo en contextos vinculados con su futura práctica profesional. El aprendizaje es experiencial ya que lo hace a través de la experiencia y reflexionando sobre la misma; aprende con otros de modo colectivo y construye un conocimiento que es compartido y socialmente distribuido, y de manera situada, ya que se desarrolla en un contexto sociocultural mediante proyectos o acciones de carácter práctico.

Este aprendizaje se desarrolla en comunidades de prácticas que, en el marco de una teoría social del aprendizaje, son un conjunto de actores sociales que comparten un objetivo común, profundizan su conocimiento y experiencia en un tema trabajando juntos e interactúan de manera continua (Wenger, 2001). Se trata de un proceso de aprender y conocer participando en comunidad. En un sentido similar, Torres define las comunidades de aprendizaje como:

"una comunidad humana organizada que construye y se involucra en un proyecto educativo y cultural propio, para educarse a sí misma, a sus niños, jóvenes y adultos, en el marco de un esfuerzo endógeno, cooperativo y solidario, basado en un diagnóstico no solo de sus carencias sino, sobre todo, de sus fortalezas para superar tales debilidades". (2001, p. 1)

En comunidad se construye un aprendizaje colaborativo. Este se concibe, desde una perspectiva sociocultural vigotskiana, como un proceso interactivo, dialógico y dialéctico donde los sujetos, que trabajan en función de una meta común, pueden tener como resultado un producto más enriquecido y acabado que si se trabajara individualmente. Implica responsabilidad individual dentro del grupo e interdependencia en un proyecto común (Zañartu Correa, 2003).

\section{Fundamentos curriculares}

Las PSC se sustentan en una concepción del curriculum como praxis orientado por un interés emancipador. Este enfoque se opone a la concepción del currículo como producto o como técnica. Su eidos orientador es la liberación de las conciencias de dependencias dogmáticas y el compromiso con la acción transformadora; la disposición que orienta la acción es la praxis, que supone una acción crítica surgida de la reflexión y dirigida a modificar deliberadamente las relaciones de poder intrínsecas a las situaciones sociales que viven las comunidades. Esto supone avanzar desde un currículo orientado a la adquisición de destrezas técnico-profesionales hacia un currículo orientado por un interés emancipador mediante la crítica ideológica o la reflexión crítica. Esto implica que las PSC, a través de la acción y la reflexión conjunta, ayuden a analizar: a) el sentido social y político de las prácticas que se realizan; b) las contradicciones existentes en ellas; c) el papel de las profesiones en la construcción y solución de los problemas de la comunidad; d) el origen o causas históricas de los problemas que se abordan; e) los intereses y supuestos ideológicos y teóricos que se expre- 
san en las situaciones; f) las posibles acciones a realizar para transformar las situaciones de injusticia, desigualdad y opresión.

Las PSC permiten incorporar la dimensión ética y crítico-social a la formación de los estudiantes para promover la conciencia social y ciudadana y la preparación para la participación social. Permiten una mayor justicia curricular ya que, siguiendo a Conell (1997), los temas curriculares se plantean y abordan desde la perspectiva o situación de los sectores más postergados o subalternizados y las profesiones se repiensan a favor de esos sectores.

El empoderamiento de las comunidades, entendiéndolo como "un proceso en el cual los profesionales trabajan compartiendo sus conocimientos con las comunidades, construyendo las instancias que permitan coadyuvar a que ellos mismos generen y construyan conocimientos como fuentes de su propio poder individual y colectivo" es un resultado esperado de las PSC (Saforcada, 2013).

\section{Concepción de universidad}

Las PSC implican una universidad comprometida y territorializada. Como se propone en el Plan Estratégico de la UNRC.

"El compromiso social de la universidad supone la democratización del conocimiento que ella produce y enseña mediante el desarrollo de prácticas académicas centradas en la intencionalidad de una mayor justicia e igualdad social. Significa, también un proceso de apropiación social y distribución de saberes que se comparten, se recontextualizan en nuevos ámbitos y se ponen al servicio de la sociedad". (2017, p. 60)

Cuando hablamos de territorialización de la universidad nos referimos al anclaje o vínculo bidireccional que ella establece con su territorio; se trata del desarrollo de actividades académicas que, en el marco del compromiso o función social de la universidad, contribuyen a la transformación de las realidades locales. Conlleva modos de colaboración, aprendizaje e intercambio entre actores institucionales y actores sociales territorialmente definidos (Juarros, 2015). Desde esta perspectiva, la universidad no está fuera del territorio sino que es una línea más en esa red de relaciones que lo configuran.

En la territorialización se borran las fronteras entre educación formal e informal, entre docencia, extensión e investigación, entre disciplinas, entre conocimiento académico y conocimiento cotidiano y popular. La red o trama territorial pasa a ser el locus de aprendizaje en las PSC.

\section{Metodología}

Como ya se anticipó, se trata de una investigación evaluativa entendida como una forma de investigación educativa que busca determinar el valor de un objeto (programa, política o institución determinada) con el fin de mejorarlo y orientar acciones futuras (Arnal, Del Rincón y Latorre, 1992).

Optamos por un enfoque interpretativo o comprensivo de investigación evaluativa que deja lugar a la consideración de los significados subjetivos que los actores otorgan, en este caso a las PSC. Comprende la situación objeto de estudio mediante la consideración de las interpretaciones, intereses y aspiraciones de los sujetos que interactúan. Reconoce multipli- 
cidad de fuentes, de criterios, de interpretaciones y de procedimientos. Se trata de utilizar una estrategia participativa de "descubrimiento de valores" acerca de lo que los docentes, estudiantes y comunidad consideran del objeto evaluado (Stake, 2006). Ofrece, además, una orientación endógena y autónoma a la evaluación al predominar una lógica emergente de criterios construidos inductivamente y desde la perspectiva de los propios actores.

Las unidades de análisis fueron 11 PSC. Los criterios para su selección fueron: a) que tuvieran continuidad desde el año 2016 hasta la actualidad; b) que estuvieran representadas las cinco facultades; c) que se contara con la posibilidad de acceder a los estudiantes para la toma de datos. Este último criterio se tuvo en cuenta puesto que en algunos casos las PSC se desarrollan en los últimos años de la carrera y los estudiantes ya no se encuentran en la universidad. A partir de estos criterios, la muestra quedó conformada según se presenta en la Tabla 1.

Tabla 1: Composición de la muestra

\begin{tabular}{|c|c|c|}
\hline Facultad & Cantidad de PSC seleccionadas & Cantidad total de PSC en desarrollo \\
\hline Ciencias Humanas & 4 & 17 \\
\hline Agronomía y Veterinaria & 3 & 12 \\
\hline $\begin{array}{l}\text { Ciencias Exactas Físico- químicas } \\
\text { y Naturales }\end{array}$ & 1 & 8 \\
\hline Ciencias Económicas & 1 & 1 \\
\hline Ingeniería & 2 & 1 \\
\hline Total & 11 & 40 \\
\hline
\end{tabular}

Fuente: elaboración propia.

Se realizaron entrevistas en profundidad a dos docentes, dos estudiantes y entre uno y dos miembros de la comunidad por cada PSC seleccionada (en total, 61 entrevistas: 24 estudiantes, 22 docentes y 15 miembros de la comunidad). Los datos se analizaron por medio de procedimientos de generación inductiva de núcleos temáticos, con un proceso analítico de codificación a través del cual el dato fue fragmentado, conceptualizado e integrado. Para eso, dicho proceso de codificación fue, en un principio, abierto, y luego se buscaron las propiedades y dimensiones de las categorías emergentes que dieron lugar a una reducción teórica en categorías centrales (Glaser y Strauss, 1967).

Se consideraron criterios de calidad del estudio como la credibilidad mediante el registro minucioso de los datos detallados, ricos y completos, y la revisión por pares (integrantes de la mesa coordinadora de PSC). Pero el más relevante, dadas las características y propósito de la investigación, fue el empoderamiento o contribución de la investigación a la implementación y mejoramiento del proyecto (Vasilachis, 2007b).

\section{Las prácticas sociocomunitarias desde la perspectiva de sus actores}

En congruencia con nuestros objetivos, analizamos a continuación las diferentes concep- 
ciones acerca de las PSC que sustentan los actores y los aportes que las mismas hacen a la comunidad.

\section{Los diferentes modos de concebir las PSC}

Un proyecto, cuando se pone en acto en el contexto de las prácticas, es interpretado y traducido por sus actores (Ball, Maguire, Braum, Hoskins \& Perryman, 2012), y una de las condiciones que media tales resignificaciones son las propias concepciones de los sujetos acerca del proyecto y su sentido. Parlett y Hamilton (1985), desde su enfoque de evaluación iluminativa, señalan que un proyecto, como "modelo abstracto", cuando se lleva a la práctica sufre modificaciones y adopta formas diferentes. $Y$ en ello incide lo que denominan el "ambiente de aprendizaje", esto es, el entorno sociopsicológico y material en el cual los participantes trabajan. En ese entorno mencionan aspectos como los presupuestos tácitos y las perspectivas de los actores intervinientes. Es por ello que en la evaluación de todo proyecto es necesario tener en cuenta tal contexto, donde las concepciones de los sujetos acerca del objeto de la innovación tienen central incidencia.

Desde las voces de los entrevistados pudimos identificar al menos dos diferentes concepciones explícitas acerca de las PSC: ${ }^{1}$ una perspectiva que podemos denominar transferencista y centrada en la formación profesional y otra que reúne rasgos de la perspectiva crítica.

\section{Perspectiva transferencista y centrada en la formación profesional}

Por un lado, se advierte en algunas expresiones una visión aplicacionista, propia de una racionalidad técnica, donde las PSC se constituyen en un contexto en el cual "aplicar" los conocimientos teóricos construidos desde la propia universidad. En ese sentido, dicen los entrevistados:

"Es un espacio donde cambia la lógica de aprender, y también bajar un montón de cosas a la práctica, a la comunidad" (E43FAYV8E) ${ }^{2}$

"Se ponen en juego muchas cosas, como la posibilidad que tienen estudiantes y docentes de acercarse al territorio y poner en práctica herramientas aprendidas desde la formación más disciplinar." (E36FCEFQyN1C)

"La interrelación con la universidad hace que ese conocimiento pueda bajar a una propuesta de mejoramiento en una ciudad. Entonces la organización xx es de alguna forma un nexo que permite esa transferencia de generación de conocimiento en la universidad a la sociedad." (E32FCEFQyN6C)

1) Aclaramos que se trata de concepciones explícitas o declarativas, ya que indagar los supuestos implícitos hubiese requerido otros procedimientos metodológicos no contemplados en este estudio.

2) La letra "E" refiere a la estrategia de recolección de datos utilizada, en este caso, entrevistas con la enumeración correspondiente. Luego colocamos las siglas de la Facultad a la que pertenece la PSC: Facultad de Agronomía y Veterinaria: FAyV; Facultad de Ciencias Económicas: FCE; Facultad de Ciencias Exactas, Físico-químicas y Naturales: FCEFQyN; Facultad de Ciencias Humanas: FCH; Facultad de Ingeniería: FI. Les sigue el número de PSC con que identificamos a cada una de las que integran la muestra y, por último, la inicial que identifica al actor entrevistado: docente: D; estudiante: E; integrante de la comunidad: C. 
Hay en los dichos de los actores una imagen topológica donde el conocimiento circula desde el lugar de la teoría (el arriba) hacia el lugar de la práctica (el abajo); circulación unidireccional de una cultura (¿concebida como superior tal vez?) que "baja" mediante operaciones de aplicación, transferencia o servicio. Subyacen a esta idea algunos rasgos de la racionalidad técnica, como son la confianza en la ciencia para la resolución de problemas prácticos y una lógica donde "los investigadores proporcionan el conocimiento básico y aplicado del que derivan las técnicas para el diagnóstico y resolución de los problemas en la práctica" (Pérez Gómez, 1988, p. 130).

Vinculadas a la anterior, están las visiones que enfatizan el aporte de las PSC en cuanto a otorgar sentido a la profesión y a la formación profesional.

"Como persona, para mí también las PSC son lugares en donde me puedo encontrar otra vez dándole valor y sentido a la profesión, el para qué elegí ser veterinaria.” (E51FAYV5D)

"Otra cosa que me parece importante de las prácticas es hacer ver a los estudiantes una realidad diferente y una oportunidad diferente. Los chicos que estudian Veterinaria van a estar muy dedicados a la producción de grandes o pequeños animales y el traerlos más al terreno y abrirles un abanico de otras oportunidades que ellos pueden ver como una salida laboral diferente." (E49FAYV4C)

"Está bueno ayudar al otro, pero yo me imaginaba eso. ¡Y nada que ver! Es una práctica de tu profesión. (E7FCH3E)

Las PSC son un espacio que permite a docentes, estudiantes y profesionales de la comunidad repensar o resignificar la profesión, darle otro sentido. Otro tipo de prácticas profesionales son posibles, desarrolladas desde otra perspectiva, en ámbitos no convencionales con un sentido social más que individual y economicista. Aquellos se repiensan a sí mismos como graduados y su futuro laboral. Las PSC se constituyen, en definitiva, como un espacio privilegiado para la formación profesional.

Desde esta óptica, el beneficio mayor parece estar en la propia universidad y en la formación profesional de sus futuros egresados, siendo las PSC un medio para tal fin. No obstante, puede advertirse un impacto social a largo plazo, en tanto estas experiencias estarían construyendo nuevas profesionalidades que es dable esperar puedan producir transformaciones con sentido crítico y social cuando se inserten en el campo laboral.

Otra idea que se reitera en el discurso de los entrevistados, tanto de los estudiantes como de los docentes, es la de las PSC como un modo de "devolución" al Estado y a la sociedad de los esfuerzos realizados en el sostenimiento de la educación universitaria pública y gratuita.

"En parte creo también que le corresponde a la universidad pública y a nosotros los formados de la universidad pública, siento que también es una manera devolver al Estado justamente con los más desfavorecidos y los más olvidados algo de todo lo que me dio el Estado a mí." (E23FCH2D)

"Yo lo hice porque, personalmente, creía que estaba bueno y aparte como que te dan ganas de ayudar por ahí, sobre todo como para devolver un poco (...) le tengo que devolver algo a la sociedad." (E35FCEFQyN1E) 
Estas visiones resaltan el carácter gratuito y público de la enseñanza universitaria sostenida por el Estado y, por lo tanto, se vivencia como un compromiso social el que la universidad realice acciones en beneficio de quien la sustenta. Sin embargo, cabe preguntarse, ¿devolución o mutuo compromiso desde diferentes ámbitos del Estado y de la sociedad (la universidad es Estado y es sociedad) por una mayor igualdad y justicia para todos?

Un actor comunitario discute esta idea sobre la "devolución" cuando dice:

"Y no es que la universidad tenga que 'devolver algo' la universidad no tiene que devolver, la universidad es un vínculo, es comunidad. Tambien es una forma de abrirse y caer en la cuenta que es algo que corresponde, para que la universidad vea eso y se genere una retroalimentación entre universidad e instituciones estatales, provinciales, nacionales, locales". (E39FCH7C)

Estas tres visiones expuestas (aplicación, formación profesional y devolución) podrían encuadrarse en lo que Cano Menoni (2017) denomina "extensionismo reformista". En ese movimiento se enlazan, a su vez, diferentes corrientes, una que advierte la extensión como un medio para una mejor formación humanista y crítica de los estudiantes y otra "cuyo énfasis estuvo puesto en dirigir la difusión de la cultura y el conocimiento universitario hacia la atención a los grandes problemas nacionales" (Cano Menoni, p. 5). Las ideas de ambas corrientes parecen tener algunos puntos en común con las concepciones antes expuestas. Se trata de perspectivas transferencistas, culturalistas, centradas en la formación profesional y a veces asistencialistas, pero que no dejan de tener un profundo sentido y valor ético.

Esta concepción predomina en docentes y estudiantes de facultades y carreras del campo de las ciencias naturales y en las de corte más profesionalista; también en miembros de la comunidad que abordan problemáticas de carácter técnico, lógica congruente con los modos de producción y distribución del conocimiento de esos campos disciplinares.

Perspectiva crítica

Otro modo de concebir a las PSC que se infiere de los testimonios de los entrevistados es una perspectiva crítica que caracterizamos a través de cuatro rasgos distintivos emergentes de los datos: interacción social, aprendizaje colaborativo, ruptura con sentido común y relación entre teoría y práctica.

En algunos testimonios prima la idea de una relación bidireccional o interacción social mutua entre la universidad y la comunidad, donde cada actor aporta lo propio.

"Cada uno aporta desde donde puede: nosotros les damos el sustento académico científico, y ellos todo lo que es social. Acá no hay plata que vaya ni que venga, es simplemente el trabajo en conjunto y la llegada social. (...) No entiendo la extensión, la transferencia, ni la PSC, si no hay un ida y vuelta. 'Puedo hacer esto por ustedes, pero ¿cuál es el resarcimiento de su parte? ¿En qué los beneficia?'. La pregunta no es ‘¿qué me dan a mí?', porque a mí no me van a dar, pero decir sí, ‘¿pudieron resolver esta situación?'. Eso es lo que yo interpreto." (E28FCEFQyN6D)

"Yo creo que la práctica sociocomunitaria por un lado es un nexo entre la universidad y lo que tiene para dar y lo que tiene para ofrecer a la comunidad en una construcción conjunta, y un análisis, una demanda que no tenga que ver con algo que la universidad quiere hacer sino con lo que la comunidad o territorio determinado necesita". (E23FCH2D) 
"La PSC es una vía para poder plantear otro montón de cosas en ese acercamiento, en ese entrecruzamiento que tiene que haber entre el mundo de afuera y la universidad, entre la universidad y la comunidad. Pero es un entrecruzamiento, porque si yo hago un acercamiento lo único que hago es acercarlo, tienen que (hace trenzas con las manos) dejarse penetrar por aquello, los programas de estudio, las prácticas institucionales, los discursos universitarios". (E22FCH2D)

"Interacción", "entrecruzamiento", "ida y vuelta", "trabajo conjunto" son los términos de los entrevistados. Ellos suponen una ruptura con la idea de transferencia lineal desde la universidad hacia la comunidad y enfatizan, en cambio, la idea de entrelazamiento entre mundo académico y mundo social, de interacción en mutuo beneficio: ambos actores reciben aportes y ambos se transforman en la articulación. Podríamos interpretar estas concepciones como acciones comunicativas en términos de Habermas (1997), esto es, situaciones donde los actores no se relacionan por un cálculo egocéntrico de utilidades e intereses sino a través del entendimiento. Los participantes en la interacción coordinan de común acuerdo sus respectivos planes de acción y los ejecutan bajo las condiciones que han establecido. Interacción comunicativa y horizontalidad como ideario y acto prevalecen en estas concepciones.

En esa interacción, los actores universitarios acompañan en la resolución de problemas.

"Es contribuir con acciones concretas para resolver situaciones. En mi caso, trabajo con problemáticas ambientales. Pero se tiene que contribuir con acciones concretas. Otra cosa en la que tenemos mucho cuidado como grupo, es plantear dos o tres acciones concretas y que se lleven adelante y se resuelvan, no plantear grandes cosas y que no se concreten. Esa es nuestra forma de movernos." (E28FCEFQyN6D)

"Me parece que hablan de eso, del compromiso social, de pertenecer a una universidad pública y poder llevar no respuestas, sino trabajar a la par a la institución. Creo que empecé a ver eso y a cambiar mi mirada al trabajo en conjunto que se hace a la par, con otras instituciones del medio." (E14FCH10E)

“Las prácticas también permiten eso de que nadie la tiene clara, nos iguala." E37FCH7D)

La idea de eficacia en las acciones como realización o concreción real para resolver los problemas subyace a los principios de actuación de un equipo. Prima la acción teleológica u orientada al logro de fines en determinada dirección como constitutiva de la acción orientada al entendimiento.

Por otro lado, la idea de acompañamiento resalta la horizontalidad de las relaciones donde los universitarios no intervienen solos ni asisten a la comunidad sino que "están junto a" ella, trabajan a la par y resuelven cooperativamente los problemas del territorio mediante acciones concretas para garantizar su efectividad.

La idea de interacción avanza en los discursos de otros actores hacia el reconocimiento de un aprendizaje colaborativo.

"Aprender de ellos y de todas estas cosas en el barrio, más que de la teoría puntualmente." (E25 FCH2E) 
"Yo no conocía absolutamente nada y yo me puse bajo el ala de $X$. Yo dije 'yo no sé nada', no dije "'yo vengo de la UNRC'... yo tengo un montón de saberes de otras cosas pero que acá no aplican." (E22FCH2D)

"Porque en esto estamos aprendiendo todos, los estudiantes, las organizaciones y los docentes." (E9FCH3D)

"Pero creo que se aprende mucho, sobre todo a trabajar con otros (...) nos obliga a repensarnos y replantearnos cosas." (E9FCH3D)

"Mi experiencia fue como un encuentro de saberes, que es más como él decía. Uno cae y es supuestamente el profesional y en realidad creo que vamos aprendiendo mutuamente. Porque cuando trabajamos con la cooperativa los chabones saben mucho de lo que ellos hacen y nosotros sabemos de lo nuestro, de la comunicación, y creo que fue eso." (E6FCH3E)

Estos testimonios de los entrevistados pueden interpretarse a la luz de lo que, en nuestro marco conceptual, referimos como comunidades de prácticas (Werger, 2001), comunidades de aprendizaje (Torres, 2001) y aprendizaje colaborativo. Los diferentes actores comparten objetivos y problemas, trabajan juntos y, mediante un esfuerzo colaborativo y solidario, aprenden y se educan en comunidad.

En las PSC, el otro me encuentra despojado de saberes pertinentes y, entonces, aprendo con él. En palabras de los mismos entrevistados, se trata de un encuentro de saberes que remite al concepto de ecología de saberes de Sousa Santos (2005). En esta perspectiva, interactúan con igual legitimidad diversos modos de conocer: conocimiento científico, cotidiano, tácito, el know how, el saber popular, la intuición, la opinión. No hay primacía de la racionalidad científica. Reconoce la pluralidad de conocimientos heterogéneos y sus interconexiones continuas y dinámicas sin comprometer su autonomía. El conocimiento es interconocimiento (Sousa Santos, 2005).

Otro componente de lo que interpretamos como un enfoque crítico es la ruptura con el sentido común que posibilitan las PSC según algunos de los actores entrevistados.

"Siempre tratamos de que sea una mirada teórica, y no desde el sentido común, no desde el prejuicio, no desde el estereotipo, que es lo que tratamos de romper desde los dos lados. Por una parte, desde algunos estudiantes que miran desde una construcción de sentido común, propio de una ubicación en una estructura de clase. Pero también tratamos de romper con una visión romántica: porque es el barrio, porque es popular está todo bien, es bueno, es lindo, tienen razón. Buscamos romper con eso también, desde las dos miradas." (E11FCH10D)

"Cómo hacer para no reforzar los prejuicios que tenemos como sujetos sociales de un sector que no habitamos. (...) No queremos que nos vengan a hablar de que la droga o que la homosexualidad son un problema biológico. No queremos. (...) Uno de los pibes dice: 'Por el hecho que yo tenga gorra, paso el andino y a mí me frenan en la heladería de enfrente a la policía'. A esto, una de nuestras estudiantes que cursó tres años de socio- 
logía, con la que trabajamos el sentido de las prácticas, le dice: ' $Y$ por qué no te sacás la gorra?'. Y el chico, un pibito de 16 años, le contesta: 'Porque si yo me saco la gorra me tengo que cambiar el color de piel, tengo que hablar de otra manera, tengo que dejar de ser yo'. Eso fue un frenadón, y ahí es cuando yo digo: no reforzar la idea de que vamos a ayudar a los pobres. Eso es muy fuerte en la carrera.» (E12FCH10D)

La ruptura con el sentido común hegemónico es posibilitada por la crítica ideológica, orientada por un interés emancipador. Para Habermas (1968), el interés emancipador supone independencia de todo lo que es externo al sujeto, liberación de dependencias dogmáticas, del sentido común hegemónico. Las ideologías tienen como una de sus funciones la naturalización o justificación del orden social existente; se reconocen en el sentido común y en el modo de ver las cosas como si se trataran de evidencias naturales. La crítica ideológica, entonces, apunta a develar lo ideológico o cultural en las prácticas sociales, a desnaturalizarlas, a poner en cuestión los significados hegemónicos y el sentido común que las impregna. Es la forma o método que permite la reflexión y la concienciación al develar cómo la ideología genera autocomprensiones distorsionadas. Se trata, según Apple, de situar los acontecimientos "en la arena, más amplia, del conflicto económico, ideológico y social" $(1979$, p. 26) buscando el origen de las creencias o del sentido común en las formas de vida social.

El último testimonio relata con claridad el modo en que el intercambio entre un estudiante y un joven de la comunidad ayuda al primero a develar su sentido común hegemónico poniendo en cuestión sus significados e interpretaciones, colocándolos en la arena de una estructura social que discrimina y estigmatiza a los sectores populares.

Ayuda a esta crítica ideológica el ver otras realidades, tener otras miradas, escuchar otras voces.

"Esto de ver y saber que nuestro contexto no es el único sino que hay un montón de otros y múltiples realidades. Y esta materia se trataba justamente de eso: poder verlo desde otra mirada, comprender eso y tratar de tener empatía." (E25FCH2E)

"No sé... no es ayuda... es escuchar las voces que están silenciadas, revertir algunas cuestiones de valores, ciertos modelos sociales, educativos y hegemónicos, siempre tuve esta capacidad para mostrar que hay otras cosas, que hay otras voces, que hay otras formas de vida, ideas, que son distintas y que no son ni mejor ni peor y que construyen subjetividad." (E22FCH2D)

La interacción con un otro que es diferente en su existencia (no en su esencia, diría Vasilachis), conocer otros mundos sociales, posibilita comprender, ponerse en el lugar del otro (empatía), entender el significado de otros valores y formas de vida y construir de ese modo subjetividades críticas.

Por último, se reitera en las entrevistas la idea de las PSC como espacio de vinculación entre teoría y práctica.

"Las definiría como el dispositivo académico que busca vincular y articular la formación de estudiantes con el territorio. Permiten potenciar la propia formación y remirar la teoría en base a esa práctica desarrollada." (E15FCH10C) 
"La PSC nos dio la posibilidad de ir al territorio y después volver al aula a poner en juego todo lo que teóricamente habíamos desarrollado o estábamos mirando desde la práctica más concreta." (E11FCH10D)

"Tenemos como una raíz desde la cátedra de trabajar siempre en esta doble mirada que son la reflexión y la acción. Se tiene que mirar dialécticamente y nosotros entendemos que para eso hace falta salir, hablar con gente, intervenir en algunas situaciones." (E12FCH10D)

La idea de praxis parece sustentar las PSC, según los entrevistados que comparten una visión crítica de la misma. Para Freire, praxis es "unidad entre práctica y teoría por la cual ambas se construyen, se formulan y reformulan en movimiento constante entre práctica y teoría para volver a una nueva práctica" (1985, p. 132). Significa unidad de la reflexión y la acción en torno a la realidad. Según el autor, a través de la praxis los hombres reconstruyen reflexivamente el mundo social, toman distancia de él y lo objetivan.

En síntesis, interacción social, comunidades de aprendizaje y de prácticas, ecología de saberes, crítica ideológica y praxis, son categorías conceptuales desde las que interpretamos los decires de los actores de las PSC. Configuran una perspectiva crítica y emancipadora que identificamos preponderantemente en docentes, estudiantes y actores sociales vinculados a la Facultad de Ciencias Humanas.

\section{Los aportes de las PSC a la comunidad}

En este apartado intentamos responder el interrogante referido a qué aportan las PSC a los actores de la comunidad desde su propia mirada. Tres categorías analíticas emergen de los datos: a) las huellas que deja la universidad; b) instalar capacidades; c) participación.

\section{Las huellas que deja la universidad}

Las PSC marcan diversas "huellas" o aportes a la comunidad desde la visión de sus actores. Podemos distinguir tres tipos de aportes o aprendizajes de la comunidad.

En primer lugar, aprendizajes de aspectos técnicos o saberes específicos que ayudaron a resolver problemas de diferente índole: de salud pública, agrícolas, botánicos, educativos, de diagnóstico y prevención, entre otros.

"Para nosotros, todo lo que sea aprendizaje para los niños es lo que más deja huella." (E27FCHC)

"Como técnica, me parece muy importante lo que se logra avanzar en cuestiones sanitarias de la provincia. Sobre todo en ámbitos donde no hay muchos recursos para comprar insumos o donde hay poca disponibilidad de trabajo humano. (...) Se hace un aporte a la salud pública en lugares donde, si no fuera por la pasantía, en muchos casos no se podría dar." (E54FAYV5C)

"Se pudo hacer un diagnóstico, una concientización de la población con la que trabajamos sobre este problema; entonces me parece que fue muy bueno. Se trabajó sobre prevención también." (E49FAYV4C) 
"Tener más cuerpos acompañando (...) al ser más personas, que es lo que aporta la práctica, nos permite ir probando distintos dispositivos alternativos al formato escolar." (E15FCH10C)

Estos aportes dejan "huellas" que admiten "despegues" que de otra manera no serían posibles, según las voces de la propia comunidad.

"Eso fue un aprendizaje que además a nosotros nos corrió el límite y nos sigue desafiando a pensar cómo seguir trabajando en ese aspecto. (...) nos desafió a seguir pensando, logramos visualizar que en realidad todavía podemos mejorar un montón." (E36FCEFQyN1C)

"El proceso que hicimos no solo que fue muy productivo, sino que hicimos un despegue que no hubiera sido posible de otra manera." (E8FCH3C)

Permiten "correr los límites" de lo que las organizaciones pueden hacer generando nuevos desafíos. Parecen actuar a modo de andamiajes que amplían las zona de desarrollo próximo (Vigotsky, 1972), es decir, la distancia entre lo que podrían hacer solos con lo que pueden hacer en colaboración con otros.

En segundo lugar, otros entrevistados valoran aportes de orden actitudinal, como aprender a integrarse y la misma convivencia humana, además de los aprendizajes técnicos.

"Hubo un montón de aprendizaje; amén de lo técnico-profesional, aprendimos que necesitamos integrarnos más. (...) Evolucionamos, crecimos, nos adaptamos, avanzamos, hicimos un montón de cosas y esto fue transformarnos, nos transformamos a nosotros mismos; entiendo que también se transformaron los estudiantes." (E8FCH3C)

"Pero hay otras cuestiones que no se miden que tienen que ver con la apertura mental, en el sentido de explorar otros mundos, creo que las prácticas en eso han aportado un montón." (E39FCH7C)

En tercer lugar, se resaltan los aportes metodológicos. La articulación con la universidad habilita nuevas metodologías y nuevas formas de resolver los problemas.

"Lo que recuperamos como positivo es que de alguna manera la PSC nos ordenó en este sentido o nos desafió a nosotros a poder pensar que eran posibles otras formas de manejar la información." (E36FCEFQyN1C)

"En el proceso nos han brindado herramientas, hemos descubierto y nos hemos vinculado con personas que nos han dado un valor agregado a la cooperativa muy importante. Nos han hecho replantear muchísimos caminos que creíamos que eran esos y no, en realidad eran otros." (E61FCHE9C)

En ese aprendizaje de nuevas metodologías, lo que a veces la universidad logra tiene que ver con hacer explícitos conocimientos que están disponibles en las organizaciones pero de manera tácita o intuitiva. Esta instancia de explicitación forma parte de un proceso de con- 
versión del conocimiento que, al formalizarlo, ordenarlo y hacerlo comunicable, lo torna más potente, transferible y eficaz.

"Yo creo que lo que hicimos, más que todo, fue facilitar o habilitar instancias, para ordenar, estructurar o perfeccionar algunas cosas que ellos de antemano sabían. (...) Lo que hicimos fue habilitar y facilitar otras instancias para que estas habilidades se potencien. (...) Desde mis prácticas, las organizaciones intuitivamente, saben más o menos lo que necesitan. Ellos saben cómo escribir o cómo manejar Instagram, pero no saben cómo hacerlo, o cómo llevar a cabo eso. Pero, ¿cómo lo hacemos? ¿Qué sería lo correcto? Esto era lo que más nos preguntaban las organizaciones." (E6FCH3E)

Además de la valoración de los aportes, resultan significativos dos testimonios donde se resalta lo que el territorio ofrece a la universidad más que lo que la universidad brinda al territorio. La comunidad resulta un "laboratorio" para el conocimiento universitario, o bien el aporte de la universidad no alcanza o no es pertinente para abordar la complejidad de los problemas del territorio.

"La granja es un laboratorio para la ciencia y para el conocimiento de hoy en la universidad." (E44FAYV8C)

"Creo que este tipo de proyectos enriquece más a la universidad que a la organización. Esto en el sentido de que para la organización supone un esfuerzo de tiempo y dedicación, y a veces las demandas de la misma son tan complejas que por ahí la estructura de la universidad, que todavía es más rígida o no le encuentra la vuelta, termina dando una primera respuesta." (E36FCEFQyN1C)

\section{Instalar capacidades}

Algunas PSC permiten no solo aprendizajes y aportes para la resolución conjunta de problemas sino que pueden avanzar en instalar capacidades en las organizaciones, instituciones o grupos, posibilitado esto además por la continuidad del trabajo conjunto.

"Para nosotros ya no son más prácticas sociocomunitarias sino un espacio más del jardín. Yo creo que nosotros no dejaríamos que lo cerraran, obviamente buscaríamos el medio, ya sea desde la universidad, del municipio, del Estado." (E27FCH2C)

"No queríamos, por insistencia de las profes, que acabe la práctica y que nosotros nos quedemos en cero. Entonces en este desarrollo pudimos ir aprendiendo algo para poder continuar con el trabajo sin que se caiga todo ese aprendizaje adquirido." (E8FCH3C)

"Hay aprendizajes que no sé si siempre se materializan en acciones pero sí se van instalando capacidades, modos distintos de hacer y de reflexionar sobre la problemática. Diría que se instalan y construyen aprendizajes en forma conjunta." (E15FCH10C)

La construcción de capacidades que quedan instaladas en la comunidad habilita a la misma a la autogestión que empodera al grupo u organización. En este sentido, Gorostiaga considera que la tarea política de la universidad consiste en "su aporte al empoderamiento cognoscitivo y actitudinal de los actores sociales, a la vez que como plataforma superior de 
aprendizaje y como conciencia crítica propositiva de la sociedad misma" (en Tünnermann, 2010, p. 27). Para Saforcada (2013) empoderamiento significa generar las condiciones para que los actores de una comunidad construyan un poder individual y colectivo que les permita tener mayor injerencia en cuestiones que hacen a sus condiciones de vida y de la sociedad toda.

La comunidad, según sus propios testimonios, se apropia de las experiencias, construye capacidades que permiten dar sustentabilidad al proyecto más allá de la permanencia o no de la universidad; esto a través de la reflexión y la acción conjunta, lo que los empodera al generar posibilidades de autonomía y autogestión.

\section{Participación}

Otro aspecto al que refieren los actores de las PSC es al alcance de su participación en el diseño, desarrollo y evaluación del proyecto.

En los dichos de uno de los entrevistados puede inferirse una participación meramente formal. Dice el actor de la comunidad:

"Hemos tenido varias experiencias de prácticas desde que empezamos con estos proyectos y siempre fue una redacción del llenado de las planillas que desde la universidad nos mandaban para poder opinar y cargar información de lo que a nosotras nos parecía necesario que estuviera presente en el documento y después se terminaba con la firma de los directivos de cada institución"." (E49FAYV4C)

"No tengo recuerdo de haber participado más que en el comentario y las devoluciones de experiencias." (E61FCHE9C)

Se trataría de una mera consulta que podría considerarse como una participación formal o simbólica que legitima decisiones no consensuadas. Siguiendo a Lucarelli (1993) y Aguerrondo (1990), se trataría de participación entendida como "formar parte de" o incorporación de personas a un grupo. Desde esta perspectiva, la participación suele ser un mecanismo para superar resistencias al cambio para dar viabilidad a proyectos verticalistas. Se le asigna un valor meramente burocrático solicitándose participación en aspectos administrativos, formales, pero no en lo político, es decir, en la toma de decisiones o formulación de objetivos.

En otros casos, podemos hablar de procesos de participación real. Los miembros de las organizaciones e instituciones participan en el diagnóstico inicial, en la identificación de problemas y casos, en el diseño y escritura del proyecto, en el armado de la estrategia de trabajo y, a veces, en la evaluación del proyecto.

"Nos estuvimos juntando con ellas (las profesoras) para escribir el proyecto. En decir que todo lo que fue el proyecto presentado formalmente nosotras tuvimos la posibilidad de agregar, sacar y darle nuestro punto de vista." (E27FCHC)

"Sí, nos sentamos con la profe en un principio para evaluar qué es lo que queríamos hacer. (...) Fuimos discutiendo casi todo punto a punto porque era lo que necesitaban tanto ellos para llevar adelante la práctica y que les sirviera a los estudiantes, como a nosotros que nos resolviera un problema puntual." (E8FCH3C) 
"El año pasado, cuando terminó el laburo a fin de noviembre, nos juntamos con el curso, evaluamos, lo charlamos, hubo una devolución, y seguimos trabajando ya que el proyecto continúa. Pero se viene dando un constante proceso de evaluación. Vemos qué venimos haciendo, cómo podríamos mejorar, qué podríamos dejar de hacer y en qué podríamos profundizar más." (E8FCH3C)

En estos casos estamos ante una participación entendida como "tomar parte en", o sea, incorporación de todas las personas en el proceso de toma de decisiones. Implica injerencia o intervención activa, acción conjunta y concertada tanto en los procesos de preparación de las decisiones como en su ejecución y evaluación. En este sentido, la participación se constituye en un mecanismo ideológico que se utiliza para transformar las relaciones sociales o relaciones de poder, donde este es compartido o distribuido entre los diferentes actores. Aquí la participación tiene un valor político o social, que es el verdadero sentido de la participación en el marco de una concepción crítica de las PSC (Lucarelli, 1993; Aguerrondo, 1990).

En síntesis, los aportes que las PSC hacen a la comunidad, que es un objetivo de las mismas, parecen ser relevantes en cuanto a los aprendizajes que dejan, resultados concretos e instalación de capacidades que fortalecen a las instituciones, organizaciones y grupos de la comunidad. No obstante, se advierten también situaciones donde el mayor beneficio lo obtiene la universidad en la formación de los estudiantes o participación simbólica de la comunidad en el diseño y evaluación del proyecto.

\section{Consideraciones finales}

Si bien este artículo presenta solo un avance parcial de una investigación mayor, podemos sintetizar algunas valoraciones y recomendaciones preliminares. El proyecto es valorado positivamente por la mayoría de los participantes. Las concepciones de los sujetos son congruentes, en la mayoría de los casos, con los principios político-ideológicos que sustentan la política institucional orientada hacia el compromiso social universitario. Sin embargo, otras concepciones, que llamamos humanistas y transferencistas, orientan acciones que modifican las intencionalidades originales y dan otro sentido a las PSC. Esto interpela a acompañar la puesta en acto del proyecto de procesos formativos colectivos que permitan explicitar, tomar conciencia y reestructurar tales concepciones.

Por otra parte, los procesos participativos y de empoderamiento de los actores, condiciones indispensables desde una perspectiva crítica de las PSC, están presentes en varias experiencias pero no en todas ellas. En especial, es necesario avanzar al respecto en las carreras vinculadas con las "ciencias duras". Este es otro aspecto a abordar mediante procesos formativos, acompañamiento a los equipos, debates permanentes y procesos de sistematización que habiliten aprendizajes colectivos.

Queda aún mucho por transitar y mejorar para acercarnos al ideal de PSC como praxis emancipadora. Ello reclama fortalecer el tejido de redes entre universidades y actores sociales, que podría ayudar en varios sentidos. Por un lado, fortaleciéndonos en el aprendizaje mutuo y la cooperación, de modo de sentar las bases para una construcción social alternativa al mundo mercantilizado que hoy nos enferma. Y por otro, creando un movimiento universitario y social unificado que interpele para que las PSC se constituyan en políticas para todo 
el sistema educativo, con presupuesto del Estado y con la evaluación y vigilancia política y epistemológica necesaria para que se sostenga el eidos emancipador.

\section{Referencias bibliográficas}

Aguerrondo, I. (1990). El planeamiento educativo como instrumento de cambio. Troquel.

Apple, M. (1979). Ideología y currículo. Akal.

Arnal, J.; Del Rincón, D. y Latorre A. (1992). Investigación educativa. Fundamentos y metodología. Labor.

Ball, S. J.; Maguire, M.; Braum, A.; Hoskins, K. \& Perryman, J. (2012). How Schools Do Policy: Policy Enactments in Secondary Schools. Taylor and Francis. Edición de Kindle.

Cano Menoni, A. (2017). La extensión universitaria y la Universidad Latinoamericana: hacia un nuevo "orden de anticipación" a 100 años de la revuelta estudiantil de Córdoba. Revista +E, 7(7), 6- 23. https://doi. org/10.14409/extension.v0i7.7047

Conell, R. W. (1997). La justicia curricular. Morata.

Freire, P. (1985). La naturaleza política de la educación. Paidós.

Glaser, B. y Strauss, A. (1967). The discovery of the grounded theory. Traducción en revisión de Pablo Rodríguez Bilella. Universidad Nacional de San Juan. Aldine Publisher.

Habermas, J. (1968). Conocimiento e interés. Taurus.

— (1997). Teoría de la Acción Comunicativa: complementos y estudios previos. Cátedra.

Heron, J. \& Reason, P. (1997). A Participatory Inquiry Paradigm. Qualitative Inquiry, 3(3), 274- 294.

Juarros, M. F. (2015). Territorialización de las prácticas académicas en la experiencia universitaria argentina. nuestrAmérica, 3(5, enero-junio). http://www.revistanuestramerica.cl/ojs/index.php/nuestramerica/article/ view/68 (11/11/2019).

Lucarelli, E. (1993). Regionalización del curriculum y capacitación docente. Miño y Dávila.

Parlett, M. y Hamilton, D. (1985). La Evaluación como lluminación. En Gimeno Sacristán, J. y Pérez Gómez, Á. (Comps.). La evaluación: su teoría y su práctica. Cuadernos de Educación № 126. Caracas.

Pérez Gómez, Á. (1988). El pensamiento práctico del profesor: implicaciones en la formación del profesorado. En Villa, A. (Coord.). Perspectivas y problemas de la función docente. Narcea.

Saforcada, E. (24 de julio de 2013). Acerca del empoderamiento en las comunidades. Conversaciones con Enrique Saforcada. Manuscrito inédito.

Sousa Santos, B. de (2005). La universidad en el siglo XXI. Para una reforma democrática y emancipadora de la universidad. Miño y Dávila Editores.

Stake, R. (2006). Evaluación comprensiva y evaluación basada en estándares. Grao.

Torres, R. M. (2001). Comunidad de aprendizaje. Repensando lo educativo desde el desarrollo local y desde el aprendizaje. Simposio Internacional sobre Comunidades de Aprendizaje. Barcelona Forum 2004.

Tünnermann, C. (2010). Prólogo. En Torres Pernalete, M. y Trápaga Ortega, M. Responsabilidad social de la universidad. Retos y perspectivas (pp. 20-27). Paidós, Tramas Sociales.

Vasilachis, I. (2007a). El aporte de la epistemología del sujeto conocido al estudio cualitativo de las situaciones de pobreza, de la identidad y de las representaciones sociales. Forum: Qualitative Social Research, 8 (3) 1-22. https://ri.conicet.gov.ar/bitstream/handle/11336/74736/CONICET_Digital_Nro.3decbefd-a736-458c-b3a2-989573128461_A.pdf

— (Coord.) (2007b). Estrategias de investigación cualitativa. Gedisa.

Vigotsky, L. (1972). Aprendizaje y desarrollo intelectual en la edad escolar. En Vigotsky, L.; Leontiev, A. y otros. Psicología y Pedagogía. Akal. 
Wenger, E. (2001). Comunidades de práctica. Aprendizaje, significado e identidad. Paidós.

Zañartu Correa, L. M. (2003). Aprendizaje colaborativo: una nueva forma de diálogo interpersonal y en red. Contexto Educativo. Revista Digital de Educación y Nuevas Tecnologías, V(28). https://s2.likedoc.org/uploads/ STE2017/04/25/WpDwNAObYk/24d828c04d99940c9ad1f043d74f0074.pdf

\section{Documentos consultados}

Universidad Nacional de Río Cuarto (2009). Resolución del Consejo Superior № 322.

(2017). Plan Estratégico Institucional 2017-2023. https://www.unrc.edu.ar/descargar/pei-2017-2023.pdf 